\title{
New Primary Vs True Recurrence In Relapsed Retroperitoneal Liposarcoma: An Analysis of Clinicopatholgical Characteristic Differences And Relapse-Free Survival
}

\section{Huan Deng ( $\sim$ dh1366053033@163.com )}

PLAGH: Chinese PLA General Hospital https://orcid.org/0000-0002-6144-2289

\section{Bo Cao}

PLAGH: Chinese PLA General Hospital

Hao Cui

PLAGH: Chinese PLA General Hospital

\section{Guibin Liu}

PLAGH: Chinese PLA General Hospital

Hanghang Li

PLAGH: Chinese PLA General Hospital

\section{Liqiang Song}

PLAGH: Chinese PLA General Hospital

\section{Ruiyang Zhao}

PLAGH: Chinese PLA General Hospital

\section{Runkai Chen}

PLAGH: Chinese PLA General Hospital

\section{Bo Wei}

PLAGH: Chinese PLA General Hospital https://orcid.org/0000-0001-7386-2689

\section{Research Article}

Keywords: retroperitoneal liposarcoma, surgery, classification, new primary, true recurrence

Posted Date: July 27th, 2021

DOl: https://doi.org/10.21203/rs.3.rs-710580/v1

License: (c) (1) This work is licensed under a Creative Commons Attribution 4.0 International License. Read Full License 


\section{Abstract}

Background This study aimed to classify relapsed retroperitoneal liposarcoma (RLS) as new primary (NP) or true recurrence (TR) and to assess the implications for therapeutic management of these classifications.

Methods Patients with recurrent RLS were classified as NP if the relapse was different from the former tumor's pathology subtype and anatomical location. Kaplan-Meier curves were adapted to estimate relapse-free survival (RFS), and logistic regression analysis was used to explore the factors related to NP.

Results Total 177 patients with relapsed RLS were included in this study. The median tumor sizes were 16 $\mathrm{cm}(\mathrm{IQR}, 13-22 \mathrm{~cm}, \mathrm{NP})$ and $18 \mathrm{~cm}(\mathrm{IQR}, 12-25 \mathrm{~cm}, \mathrm{TR})(\mathrm{P}=0.003)$. Multifocal tumors (89.2\% vs $73.8 \%$, $\mathrm{P}=0.011)$ and multiple pathology subtypes $(52.7 \%$ vs $31.1 \%, \mathrm{P}=0.004)$ were more common in the NP group and tended to invade wider anatomical areas ( $85.1 \%$ vs $71.8 \%, P=0.037)$. The median RFS was 17 months (IQR, 7-35 months) in the NP group and 12 months (IQR, 5-23 months) in the TR group, and NP patients showed a longer RFS than TR patients $(P=0.004)$. When the log-rank test was conducted, lowgrade pathology, tumor growth rate $\leq 1.25 \mathrm{~cm} /$ month and tumor size $\leq 16.5 \mathrm{~cm}$ had a significant influence on the NP phenomenon $(P=0.015,0.019$, and 0.028 , respectively). Logistic regression analysis illustrated that current surgeries, pathology subtype varieties and pathology grade were independent risk factors for NP ( $P=0.017,0.019$, and 0.025 , respectively).

Conclusion NP patients have longer RFS than TR patients, and their tumors tend to have multiple pathology subtypes and tumors and are more likely to invade wider anatomical areas. This classification contributes to a better understanding of RLS and provides new evidence for different therapeutic management of relapsed tumors.

\section{Introduction}

Retroperitoneal liposarcoma (RLS) is a rare malignant tumor arising in the retroperitoneum and is the most common type of retroperitoneal sarcoma. RLS accounts for $0.07-0.2 \%$ of all tumors and approximately $12 \%$ and $40 \%$ of all liposarcomas ${ }^{[1]}$. This tumor type can be further divided into 4 subtypes in the updated WHO (World Health Organization) classification: well-differentiated liposarcoma (WDL), dedifferentiated liposarcoma (DDL), myxoid cell liposarcoma (MLS), and pleomorphic liposarcoma (PLS). WDL/DLL is the major subtype of retroperitoneal liposarcoma, accounting for approximately $56 \% / 37 \%$ of primary retroperitoneal liposarcoma, according to a related report ${ }^{[2]}$. The prognosis of RLS is correlated with the pathological type, and high-grade tumors are associated with high rates of local recurrence and distant metastasis ${ }^{[3]}$. RLS has relapse propensity after surgery, which is the main treatment for this disease at present ${ }^{[4]}$. The resection margin of retroperitoneal sarcoma and histology subtype remain the most important prognostic predictors of local recurrence and overall survival $[2,5,6]$. 
Numerous studies have demonstrated that combined resection of adjacent organs such as the kidney and gastrointestinal tissues in the abdomen will improve the local outcome. However, the large tumor size and complicated anatomic structures of RLS sometimes limit the ability of the surgeon to achieve negative surgical margins and are predictive factors of poor prognosis ${ }^{[7]}$. Complete capsular resection and combined organs resection rarely achieve radical cure of RLS, which is currently a challenge ${ }^{[8]}$. RLS relapse after surgical resection typically appears as recurrence or a locoregional pattern ${ }^{[9,10]}$. However, we found that some cases of relapsed RLS were distinct from traditional recurrence. As the number of relapse events increases, the location of the tumor may shift but not metastasize in the retroperitoneum, indicating the possibility of a different relapse mechanism in RLS. However, no reliable guidelines or study has defined the subset of patients.

We aimed to analyze the basic clinical and pathological characteristics of recurrent RLS patients who had undergone multiple surgeries at our center. We evaluated two decades of RLS cases in our department and proposed a novel relapse phenomenon intended to reveal that a subset of relapsed tumors of RLS is new primary (NP) and originates in different new-onset locations ${ }^{[11]}$. This classification contributes evidence for different therapeutic management strategies of RLS. We believe TR and NP may have different natural histories, different prognoses, and different clinical behavior.

\section{Materials And Methods Patient selection}

The cases were selected from the medical record management system of the Chinese People Liberation Army General Hospital from February 2000 to February 2020. All patients experienced resurgery within 5 years after relapse, and the resurgery medical records were applied to this study. Their pathology was diagnosed and confirmed by experienced pathologists based on WHO criteria (WDL, DDL, MLS, PLS) ${ }^{[12]}$. Other types of retroperitoneal tumors were excluded from this study. Patients were also excluded from the study if they had distant organ metastasis or extensive abdominal metastasis. Patients treated with adjuvant radiotherapy or chemotherapy were also not enrolled. Patients who relapsed but did not undergo resurgery were excluded. The diagnosis of relapse RLS combined preoperative radiological examinations (computed tomography (CT), magnetic resonance imaging (MRI), ultrasound), postoperative pathological examinations and operation dictations.

\section{Definition}

Recurrent retroperitoneal liposarcoma was defined as tumors that relapsed multiple times from the initial diagnosis with at least two surgeries. To describe the tumor location, we divided the retroperitoneum and pelvic cavity into seven compartments (Fig. 1). A unifocal tumor was defined as the presence of one solitary neoplasm, and a multifocal tumor was defined as the presence of two or more noncontiguous neoplasms. True recurrence (TR) was defined as tumor relapse at the same anatomical area with the same pathological subtype (such as WDL to WDL) after surgery. A new primary tumor (NP) was defined 
as tumor relapse to another subtype after R0 resection (such as WDL to DLS), and the tumor's center location changed to another compartment (such as "1" to "4") (Fig. 1). Relapse-free survival (RFS) was defined as the time from surgery to the time of relapse diagnosis ${ }^{[13]}$.

\section{Statistical Analysis}

The statistical analysis excluded cases with incomplete records, and the main statistical tool was SPSS 26.0 software. Categorical and quantitative data were compared using the chi-square or Fisher exact test and the Mann-Whitney U-test, respectively. Kaplan-Meier curves were used to estimate the RFS of different groups, and logistic regression analysis was used to explore the risk factors for NP. All the tests were two-sided, and a $\mathrm{P}$ value $<0.05$ was considered statistically significant.

\section{Results}

\section{Patient Characteristics}

We identified 201 patients with recurrent RLS; among them, 24 patients relapsed after surgery but did not undergo resurgery or had incomplete clinical data. Finally, 177 patients (100 men and 77 women) were enrolled in the study. According to the above screening criteria, 74 patients were classified as NP, and 103 patients were classified as TR. In the NP and TR groups, the proportion of men (62.2\% vs $52.4 \%$ ) was larger than that of women, and the mean ages were 52 and 53 years old, respectively. The distribution of clinical and pathological characteristics among patients is illustrated in Table 1. 
Table 1

Characteristics of two relapsed groups

\begin{tabular}{|c|c|c|c|}
\hline & $\begin{array}{l}\text { New primary }(n= \\
\text { 74) }\end{array}$ & True recurrence $(n=103)$ & P Value \\
\hline Mean age at initial diagnosis, years & $47 \pm 10.6$ & $49 \pm 10.8$ & 0.540 \\
\hline Mean age at this relapse, years & $52 \pm 11.4$ & $53 \pm 10.6$ & 0.838 \\
\hline \multicolumn{4}{|l|}{ Sex0.198 } \\
\hline male, n (\%) & $46(62.2)$ & $54(52.4)$ & \\
\hline female, n (\%) & $28(37.8)$ & $49(47.6)$ & \\
\hline ASA & & & 0.302 \\
\hline$\leq 2, \mathrm{n}(\%)$ & $54(73.0)$ & $82(79.7)$ & \\
\hline$>2, \mathrm{n}(\%)$ & $20(27.0)$ & $21(20.3)$ & \\
\hline Tumor size (cm), median (IQR) & $16(13-22)$ & $18(12-25)$ & 0.003 \\
\hline Current relapse time & & & 0.021 \\
\hline 2nd surgeries, n (\%) & $33(44.6)$ & $64(62.1)$ & \\
\hline >2nd surgeries, $\mathrm{n}(\%)$ & $41(55.4)$ & $39(37.9)$ & \\
\hline $\begin{array}{l}\text { Growth rate (cm/month), median } \\
\text { (IQR) }\end{array}$ & $1.09(0.52-2.60)$ & $1.29(0.68-2.65)$ & $<0.001$ \\
\hline \multicolumn{4}{|l|}{ Tumor numbers 0.011} \\
\hline unifocal & $8(10.8)$ & $27(26.2)$ & \\
\hline multifocal & 66(89.2) & $76(73.8)$ & \\
\hline Tumor capsular completeness, $\mathrm{n}(\%)$ & & & 0.500 \\
\hline complete & $44(59.5)$ & $56(54.4)$ & \\
\hline incomplete & $30(40.5)$ & $47(45.6)$ & \\
\hline Resection completeness, n (\%) & & & 0.643 \\
\hline complete & 63(85.1) & $85(82.5)$ & \\
\hline incomplete & 11(14.9) & 18(17.5) & \\
\hline Organs combined resection & & & 0.793 \\
\hline yes & $44(79.7)$ & $65(73.8)$ & \\
\hline no & $30(20.3)$ & $38(26.2)$ & \\
\hline Important vessels invaded & & & 0.819 \\
\hline
\end{tabular}




\begin{tabular}{|c|c|c|c|}
\hline & $\begin{array}{l}\text { New primary }(n= \\
74)\end{array}$ & True recurrence $(n=103)$ & P Value \\
\hline yes & $44(79.7)$ & $63(61.2)$ & \\
\hline no & $30(20.3)$ & $40(38.8)$ & \\
\hline \multicolumn{4}{|l|}{ Tumor invaded regions 0.037} \\
\hline$\leq$ two regions & $11(14.9)$ & $29(28.2)$ & \\
\hline$>$ two regions & $63(85.1)$ & 74(71.8) & \\
\hline Bleeding volume (ml) & $1000(500-1950)$ & $1000(500-2000)$ & 0.951 \\
\hline Blood transfusion & & & 0.359 \\
\hline yes & $59(79.7)$ & $76(73.8)$ & \\
\hline no & $15(20.3)$ & $27(26.2)$ & \\
\hline Length of inpatient time (days) & $18(15-28)$ & $19(15-25)$ & 0.722 \\
\hline Pathology subtype varieties & & & 0.004 \\
\hline single pathology & $35(47.3)$ & $71(68.9)$ & \\
\hline multiple pathology & $39(52.7)$ & $32(31.1)$ & \\
\hline Pathology grade & & & 0.108 \\
\hline high & $37(50.0)$ & $64(62.1)$ & \\
\hline low & $37(50.0)$ & $39(37.9)$ & \\
\hline
\end{tabular}

\section{Clinicopathological features of two groups}

We analyzed the clinicopathological characteristics of patients with NP tumors and compared them with those of conventional TR tumors. According to the screening criteria above, 74 patients displayed the NP phenomenon, and 103 patients were enrolled in the TR group. In the NP and TP groups, the median tumor size was $16 \mathrm{~cm}(\mathrm{IQR}, 13-22 \mathrm{~cm})$ vs $18 \mathrm{~cm}(\mathrm{IQR}, 12-25 \mathrm{~cm})$, respectively $(P=0.003)$, indicating that the tumor burden of TR patients was heavy. The tumor invaded the area in the abdomen beyond two regions, and the NP group tended to invade a wider anatomical area $(85.1 \%$ vs $71.8 \%, P=0.037)$. This characteristic is closely associated with tumor numbers. Multifocal tumors were more common in the NP group ( $89.2 \%$ vs $73.8 \%, \mathrm{P}=0.011)$. In addition, NP tumors generally had multiple pathology subtypes $(52.7 \%$ vs $31.1 \%, P=0.004)$. The tumor number and tumor pathology subtype varieties showed remarkable differences between the two groups. The median tumor growth rate was lower in the NP group $(1.09 \mathrm{~cm} /$ month) than in the TR group $(1.29 \mathrm{~cm} /$ month), but the difference was insignificant $(p=$ 0.286). Pathology grade was also more common in the NP group, but the difference was statistically insignificant $(50.0 \%$ vs $37.9 \%, P=0.108)$. Current surgeries were an important variable related to 
prognosis, and most TR patients exceeded two surgeries in this study ( $55.4 \%$ vs $37.9 \%, P=0.021)$. The other surgery-related variables between NP and TR are also illustrated in Table 1.

\section{Relapse-free survival (RFS)}

The median RFS was 17 months (IQR, 7-35 months) in the NP group and 12 months (IQR, 5-23 months) in the TR group, and the NP group showed a longer RFS than the TR group (Fig. 2). When the log-rank test was conducted, it found that low-grade pathology, tumor growth rate $\leq 1.25 \mathrm{~cm} / \mathrm{month}$ and tumor size $\leq$ $16.5 \mathrm{~cm}$ were significant influencing factors of the NP phenomenon $(P=0.015,0.019$, and 0.028 , respectively) (Figs. 3-5). Logistic regression analysis illustrated that current surgeries, pathology subtype varieties and pathology grade were independent risk factors for NP $(P=0.017,0.019$, and 0.025 , respectively) (Table 2 ). 
Table 2

Logistic regression analysis of the risk factors for new primary tumors

\begin{tabular}{|c|c|c|c|c|c|c|}
\hline \multirow{2}{*}{$\begin{array}{l}\text { Variables } \\
\text { Pathology grade } \\
\text { (high vs low) }\end{array}$} & \multirow{2}{*}{$\begin{array}{l}\boldsymbol{\beta} \\
0.420\end{array}$} & \multirow{2}{*}{$\begin{array}{l}\text { Standard } \\
\text { Error } \\
0.330\end{array}$} & \multirow{2}{*}{$\begin{array}{l}\text { Odds } \\
\text { Ratio } \\
1.522\end{array}$} & \multicolumn{2}{|c|}{$\begin{array}{l}95 \% \text { Confidence } \\
\text { Interval }\end{array}$} & \multirow{2}{*}{$\begin{array}{l}P \\
0.203\end{array}$} \\
\hline & & & & 0.797 & 2.905 & \\
\hline $\begin{array}{l}\text { Resection completeness } \\
\text { (complete vs incomplete) }\end{array}$ & -0.276 & 0.371 & 0.759 & 0.367 & 1.570 & 0.457 \\
\hline $\begin{array}{l}\text { Tumor growth rate } \\
\text { (cm/month) } \\
(\leq 1.25 \text { vs }>1.25)\end{array}$ & -0.580 & 0.361 & 0.560 & 0.276 & 1.137 & 0.108 \\
\hline $\begin{array}{l}\text { Tumor size }(\mathrm{cm}) \\
(\leq 16.5 \mathrm{vs}>16.5)\end{array}$ & -0.463 & 0.365 & 0.629 & 0.308 & 1.286 & 0.204 \\
\hline $\begin{array}{l}\text { Current surgery time } \\
\text { ( } \leq \text { 2nd vs }>2 \text { nd) }\end{array}$ & 0.680 & 0.329 & 1.973 & 1.035 & 3.763 & 0.039 \\
\hline $\begin{array}{l}\text { Pathological subtype variety } \\
\text { (single vs multiple) }\end{array}$ & 0.689 & 0.334 & 1.993 & 1.036 & 3.834 & 0.039 \\
\hline $\begin{array}{l}\text { Tumor invaded region } \\
\text { ( } \leq \text { two regions vs > two } \\
\text { regions) }\end{array}$ & 0.940 & 0.435 & 2.560 & 1.091 & 6.010 & 0.031 \\
\hline $\begin{array}{l}\text { Tumor number } \\
\text { (unifocal vs multifocal) }\end{array}$ & 0.695 & 0.470 & 2.003 & 0.797 & 5.031 & 0.139 \\
\hline $\begin{array}{l}\text { Tumor capsular } \\
\text { completeness } \\
\text { (complete vs incomplete) }\end{array}$ & -0.290 & 0.352 & 0.748 & 0.376 & 1.491 & 0.410 \\
\hline
\end{tabular}

\section{Discussion}

In this study, we attempted to classify all patients experiencing RLS relapse at our hospital as either new primary or true recurrence and to explore the prognostic and therapeutic significance of these classifications. We investigated the baseline and clinicopathological characteristics of 177 recurrent RLS patients. Men were more susceptible to this disease, and 52-53 years was the pivotal age in this study. Patients with NP relapse were younger at initial diagnosis and current relapse than those who experienced TR, but there were no significant differences. Our study demonstrated that $41.8 \%$ of RLS cases were NP tumors based on changes in anatomical location and histology subtype. We found that 
the rate of relapse was more common in the TR style (58.2\%), but the NP style had certain features that deserve particular attention. The median RFS was 17 months (IQR, 7-35 months) in the NP group and 12 months (IQR, 5-23 months) in the TR group, and the NP group showed a longer RFS than the TR group (Fig. 2). In other words, TR has a higher relapse rate and shorter relapse interval, which contributes evidence for different management strategies between NP and TR. TR patients should receive a shorter follow-up interval during clinical management than NP patients.

We divided recurrent RLS into NP and TR groups according to the above classification standard. NP indicates that the tumor's pathological components change into different subtypes and that the center location changes into other anatomical compartments after RO resection. First, the finding that the RLS subtype has a core effect on prognosis is widely accepted at present $[6,15,16]$. Some studies have demonstrated that the four subtypes showed different typical gene aberrations: WDL shows MDM2, CDK4, HMGA2, FRS2, DDR 2, PPAR- $y$, and YEATS4 aberrations; DDL shows FGFR3, RB, and CEBPA aberrations; MLS shows RET and FUS-DDIT3 aberrations; and PLS shows P53 and VEGF aberrations ${ }^{[3]}$. The cytogenetic signature of WDL displays amplified sequences from the long arm of chromosome 12 (12q13-15); DDL has common genes with WDL but with more complexity ${ }^{[17,18]}$. MLS has a gene alteration in FUS-DDIT3 at t(12;16) (q13; p11) ${ }^{[19]}$. WDL and DDL represent typical low-grade and highgrade RLS, respectively. At the level of cytogenetic alteration, the four RLS subtypes have different characteristics. Thus, pathological subtype alterations during the relapse process deserve increased attention because their biological behavior and clinical characteristics are not similar. Poor dedifferentiation has previously been shown to coincide with a decrease in DFS and OS in RLS ${ }^{[20,21]}$. In regard to the further impact of low grade on relapse, there are few studies to illustrate whether low grade is different for certain special relapses. In our study, we found that low-grade RLS had an obvious difference between NP and TR, and NP patients tended to have a longer RFS than TR patients (Fig. 3). However, high-grade RLS did not have a specific influence on RFS among the two groups $(P=0.114)$. We wondered whether the underlying mechanism was associated with NP pathogenesis. Additionally, we found that NP patients tended to have multiple tumors (89.2\%), which is lateral proof of NP. During longterm clinical practice, we believe that some tumors are latent or that microscopic status cannot be perceived at the retroperitoneal regions when we conduct resection for large tumors. These tumors tend to be ignored and grow rapidly after surgery, showing a short-term recurrence phenomenon. However, some of these tumors are new primary tumors because the pathology and tumor center location are completely different from those of the former tumor. When latent tumors develop enough volume to be examined, new relapsed RLS is established. In addition, we found that most NP patients had multiple pathology subtypes in this study $(52.7 \%)$, which is a corresponding manifestation of tumor numbers in the NP group. Therefore, NP tumors were more likely to invade wider anatomical compartments in the abdomen $(P=0.037)$. The overall median tumor growth rate in NP and TR was $1.25 \mathrm{~cm} / \mathrm{month}$, and it was different between NP and TR $(1.09 \mathrm{~cm} /$ month vs $1.29 \mathrm{~cm} /$ month, $\mathrm{P}<0.001)$. James 0 . Park et al demonstrated that a tumor growth rate greater than $0.9 \mathrm{~cm} /$ month was associated with poor outcomes ${ }^{[2]}$. In our study, we found that a rate $\leq 1.25 \mathrm{~cm} /$ month was beneficial for NP patients $(P=0.019)$, but the RFS of the two groups was not different when the growth rate was $>1.25 \mathrm{~cm} / \mathrm{month}$ (Fig. 4). We 
hypothesize that the phenomenon is due to certain abnormal signs that the tumor volume induces when the growth rate is $>1.25 \mathrm{~cm} /$ month. Patients perceive it to be uncomfortable and go to the hospital, and thus RFS is reached at that point. We also found some differences in the risk factor profile for patients experiencing NP relapse and those experiencing true recurrence by logistic regression analysis. We demonstrated that current surgeries $(P=0.039)$, tumor invaded regions $(P=0.031)$ and pathological subtype varieties $(P=0.039)$ were independent risk factors for new primary tumors (Table 2$)$.

NP and TR show differences in many aspects, from the cytogenetic mechanism to clinical characteristics, factors reflecting that therapeutic management should adapt different strategies between TR and NP. Presently, surgery remains the most effective treatment, and conducting complete or aggressive bloc resection is considered necessary under certain circumstances. Whether it is necessary to conduct a different surgery strategy among NP and TR lacks in-depth research. RLS tumors tended to invade more important blood vessels and organs, particularly in the NP group (Table 1). This feature may be correlated with tumor numbers and tumor-involved regions. If a patient has multiple tumors, the scope of resection will definitely increase. RLS is a complicated disease, and the bleeding volume is the typical reflection variable, which reveals that this disease is difficult to cope with. In our study, more than half of the patients in every surgery group had a bleeding volume exceeding $1000 \mathrm{ml}$ (Table 1), and their postoperative hospital stay time generally lasted approximately 20 days. Tumor size is another special feature of RLS, and the median tumor size reached $16 \mathrm{~cm}$ in the NP group and $18 \mathrm{~cm}$ in the TR group. This characteristic usually makes surgery more difficult because of the important organs and vessels located in the abdomen. Sometimes, adopting an intentional incomplete resection strategy is beneficial for the patient's prognosis ${ }^{[5,22]}$, but the cost is inevitable recurrence ${ }^{[23]}$. Therefore, achieving an absolute negative resection margin during surgery is difficult under some circumstances. However, following bloc resection or macroscopic negative margin therapy, some patients exhibit short-term relapse. Surprisingly, even with a microscopic negative resection margin, some RLS may still relapse in a short time. Thus, we speculate that some of the relapsed tumors were different from those with conventional true recurrence. In our results, TR had a shorter RFS, a rapid tumor growth rate, and was more likely to result in short-term relapse after surgery. According to past studies, RLS recurrence is quickly often related to incomplete resection margins and poor histological subtypes ${ }^{[5,15]}$. Consistent with our study, TR tumors usually originate in remnant tumors, relapse in the same pathology subtype of former tumors, and relapse at a rapid speed. Nevertheless, differences among TR and NP are still unclear at present, and further proof concerning pathogenesis, prognoses, and survival remains ambiguous because of the study limitations, thus requiring a large number of cases and multicenter cooperation, as well as more basic studies to explore the underlying mechanism ${ }^{[24]}$.

Our study has several limitations. First, this study involved a single institution, and the number of cases was small. Second, the long-term overall survival for recurrent RLS should be evaluated. Third, our cases were retrospective, mainly based on our institution's medical records, examination records and pathology reports, and lacked prospective data. Fourth, the data for some factors, such as tumor necrosis and the mitotic count, were missing, making pathological grading difficult. 


\section{Conclusion}

In conclusion, RLS is a recurrent disease, and surgery is currently the most effective treatment. Some relapse tumors of RLS appear to present a neoplasm pathogenesis mechanism, originating at a different new-onset point and exhibiting certain natural histories. Classifying those tumors as NP contributes to a better understanding of RLS and gives them different therapeutic management. NP patients have longer RFS than TR patients, and their tumors tend to have multiple pathology subtypes and tumors and are more likely to invade wider anatomical areas. Concerning other differences, such as prognosis, the overall survival and underlying pathogenesis mechanism need more clinical and basic research. This classification contributes to a better understanding of RLS relapse and provides new evidence for different therapeutic management strategies.

\section{Abbreviations}

RLS, retroperitoneal liposarcoma; NP, new primary; TR, true recurrence; WDL, well-differentiated liposarcoma; DDL, dedifferentiated liposarcoma; MLS, myxoid cell liposarcoma; PLS, pleomorphic liposarcoma; WHO, World Health Organization; IQR interquartile range.

\section{Declarations}

\section{Ethics Approval and Informed Consent}

The institutional review board of the institutional research ethics committee of the Chinese PLA General Hospital approved this retrospective study in 2020.

\section{Consent for Publication}

All authors have read and approved this manuscript. All authors consent to the publication of this article

\section{Acknowledgments}

We would like to thank colleagues from Chinese PLA General Hospital for their valuable assistance in the statistical analysis and data collection.

\section{Authors' Contributions}

All authors contributed to data collection, statistics, drafting or amending the paper, have agreed on the journal to which the article will be reviewed, gave their final approval of the version to be published, and agree to be responsible for all aspects of the work.

\section{Funding}

This project was supported by grants from the National Natural Science Foundation of China (81773135) and National Key Research and Development Project of China (2019YFB1311505). 


\section{Disclosure}

The authors report no conflicts of interest in this work.

\section{References}

[1] Adarsh V, Lakshmi R. Retroperitoneal liposarcoma: a comprehensive review.[J]. American journal of clinical oncology,2013,12(4):5665-5667.

[2] Park J O, Qin L, Prete F P, et al. Predicting Outcome by Growth Rate of Locally Recurrent Retroperitoneal Liposarcoma[J]. Annals of Surgery,2009,250(6):977-982.

[3] Tyler R, Wanigasooriya K, Taniere P, et al. A review of retroperitoneal liposarcoma genomics[J]. Cancer Treatment Reviews,2020,86:102013.

[4] Trans-Atlantic R S W G. Management of metastatic retroperitoneal sarcoma: a consensus approach from the Trans-Atlantic Retroperitoneal Sarcoma Working Group (TARPSWG)[J]. Annals of oncology,2018,29(4):857.

[5] Singer S, Antonescu C R, Riedel E, et al. Histologic Subtype and Margin of Resection Predict Pattern of Recurrence and Survival for Retroperitoneal Liposarcoma[J]. Annals of Surgery,2003,121:52-65.

[6] Tan M C B, Brennan M F, Kuk D, et al. Histology-based Classification Predicts Pattern of Recurrence and Improves Risk Stratification in Primary Retroperitoneal Sarcoma[J]. Annals of Surgery,2016,263(3):593-600.

[7] Strauss D C, Hayes A J, Thway K, et al. Surgical management of primary retroperitoneal sarcoma[J]. $\mathrm{Br}$ J Surg,2010,97(5):698-706.

[8] Lee S Y, Goh B K P, Teo M C C, et al. Retroperitoneal liposarcomas: the experience of a tertiary Asian center[J]. World journal of surgical oncology,2011,9(1):12.

[9] Tseng W W, Madewell J E, Wei W, et al. Locoregional Disease Patterns in Well-Differentiated and Dedifferentiated Retroperitoneal Liposarcoma: Implications for the Extent of Resection?[J]. Annals of Surgical Oncology,2014,21(7):2136-2143.

[10] Bagaria S P, Gabriel E, Mann G N. Multiply recurrent retroperitoneal liposarcoma[J]. Journal of Surgical Oncology,2018,117(1):62-68.

[11] Smith T E, Lee D, Turner B C, et al. True recurrence vs. new primary ipsilateral breast tumor relapse: an analysis of clinical and pathologic differences and their implications in natural history, prognoses, and therapeutic management[J]. Int J Radiat Oncol Biol Phys,2000,48(5):1281-1289. 
[12] Jo V Y, Fletcher C D M. WHO classification of soft tissue tumors: an update based on the 2013 (4th) edition[J]. Pathology,2014,46(2):95-104.

[13] Ishii K, Yokoyama Y, Nishida Y, et al. Characteristics of primary and repeated recurrent retroperitoneal liposarcoma: outcomes after aggressive surgeries at a single institution[J]. Japanese Journal of Clinical Oncology,2020.

[14] Bartlett E K, Curtin C E, Seier K, et al. Histologic Subtype Defines the Risk and Kinetics of Recurrence and Death for Primary Extremity/Truncal Liposarcoma[J]. Annals of Surgery,2020:1.

[15] Gronchi A, Strauss D C, Miceli R, et al. Variability in Patterns of Recurrence After Resection of Primary Retroperitoneal Sarcoma (RPS)[J]. Annals of Surgery,2016,263(5):1002-1009.

[16] Beird H C, Wu C, Ingram D R, et al. Genomic profiling of dedifferentiated liposarcoma compared to matched well-differentiated liposarcoma reveals higher genomic complexity and a common origin[J]. Molecular Case Studies,2018,4(2):a2386.

[17] Thway K. Well-differentiated liposarcoma and dedifferentiated liposarcoma: An updated review[J]. Seminars in Diagnostic Pathology,2019,36(2):112-121.

[18] Sbaraglia M, Dei Tos A P. The pathology of soft tissue sarcomas[J]. La radiologia medica,2019,124(4):266-281.

[19] Jm S, S R, M D, et al. Risk factors of early recurrence in retroperitoneal liposarcoma.[J]. Cirugia espanola,2018,96(9):568-576.

[20] Keung E Z, Hornick J L, Bertagnolli M M, et al. Predictors of Outcomes in Patients with Primary Retroperitoneal Dedifferentiated Liposarcoma Undergoing Surgery[J]. Journal of the American College of Surgeons,2014,218(2):206-217.

[21] Shibata D. Is there a role for incomplete resection in the management of retroperitoneal liposarcomas?[J]. Journal of the American College of Surgeons,2001,193(4):373-379.

[22] Kirane A, Crago A M. The importance of surgical margins in retroperitoneal sarcoma[J]. J Surg Oncol,2016,113(3):270-276.

[23] Wang J, Grignol V P, Gronchi A, et al. Surgical management of retroperitoneal sarcoma and opportunities for global collaboration[J]. Chinese clinical oncology,2018,7(4):39.

\section{Figures}




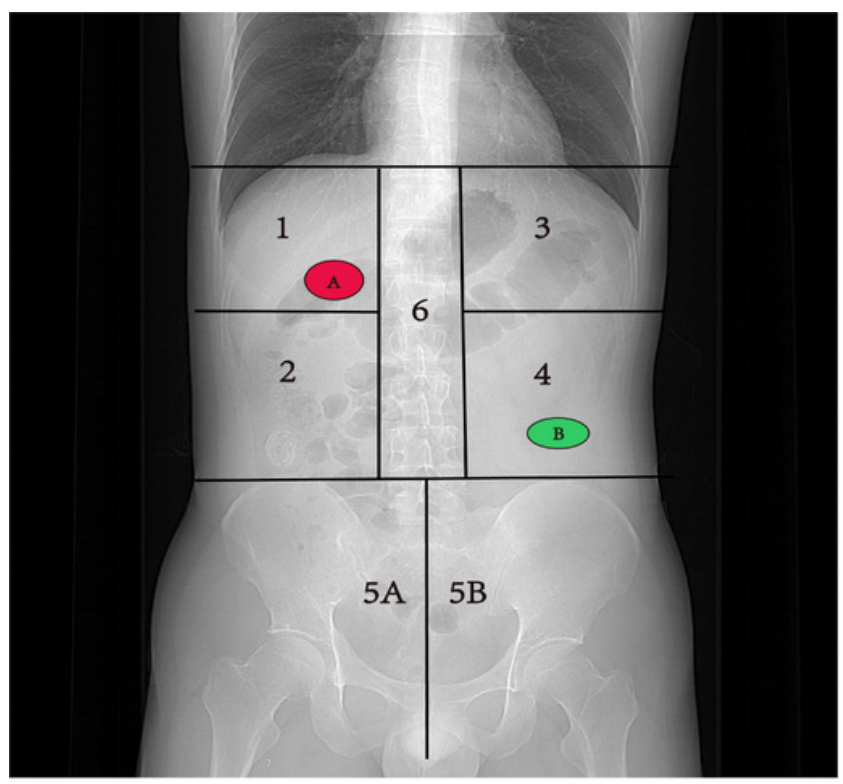

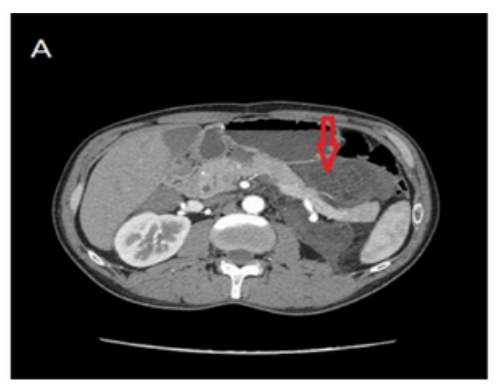

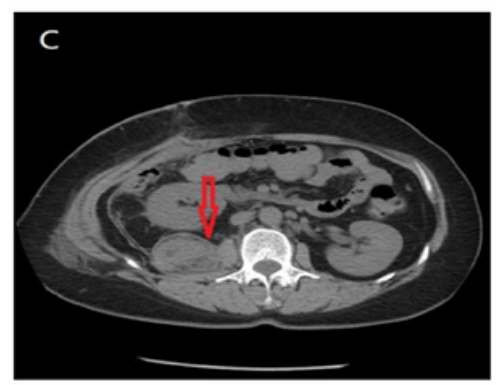

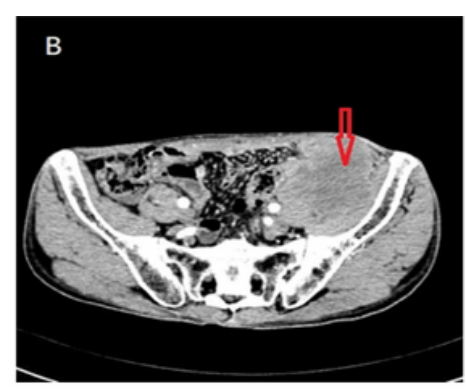

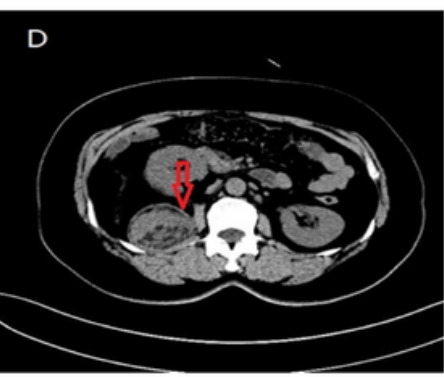

\section{Figure 1}

a. Tumor location in the abdominal cavity. The abdomen and pelvic areas are divided into seven compartments. Three horizontal lines represent the diaphragm level, L2 or renal $(\mathrm{a}, \mathrm{v})$ level, and pelvic brim level, respectively. Vertical lines represent two sides and midline of the spine, forming 4 abdominal compartments $(1,2,3,4) .5 \mathrm{~A}$ and $5 \mathrm{~B}$ represent the perinephric area, and area 6 is the abdominal aorta and inferior venous cava area. The red A ellipse represents a tumor occurring in compartment " 1 " in a patient, and the green $B$ ellipse represents a relapsed tumor occurring in another compartment after the red $A$ tumor has been resected during surgery. Green $B$ indicates the location and pathology transfer compared to red $A$ (green $B$ is a new primary tumor compared to the red $A$ tumor in a patient). $b$. The CT scan of two relapsed RLS. A and B comprise a new primary group. A, B are two examples of continuous RLS disease of in a patient; A: a low-grade tumor situated at area " 1 " that experienced complete resection; $B$ : a high-grade tumor occurring at " $5 \mathrm{~B}$ " area after $A$ resection. $C$ and $D$ comprise a true-recurrence group. $\mathrm{C}$ and D are two continuous RLS diseases in a patient; C: a high-grade tumor located in area "2" experienced complete resection; D: a high-grade recurrent tumor in area "2". 


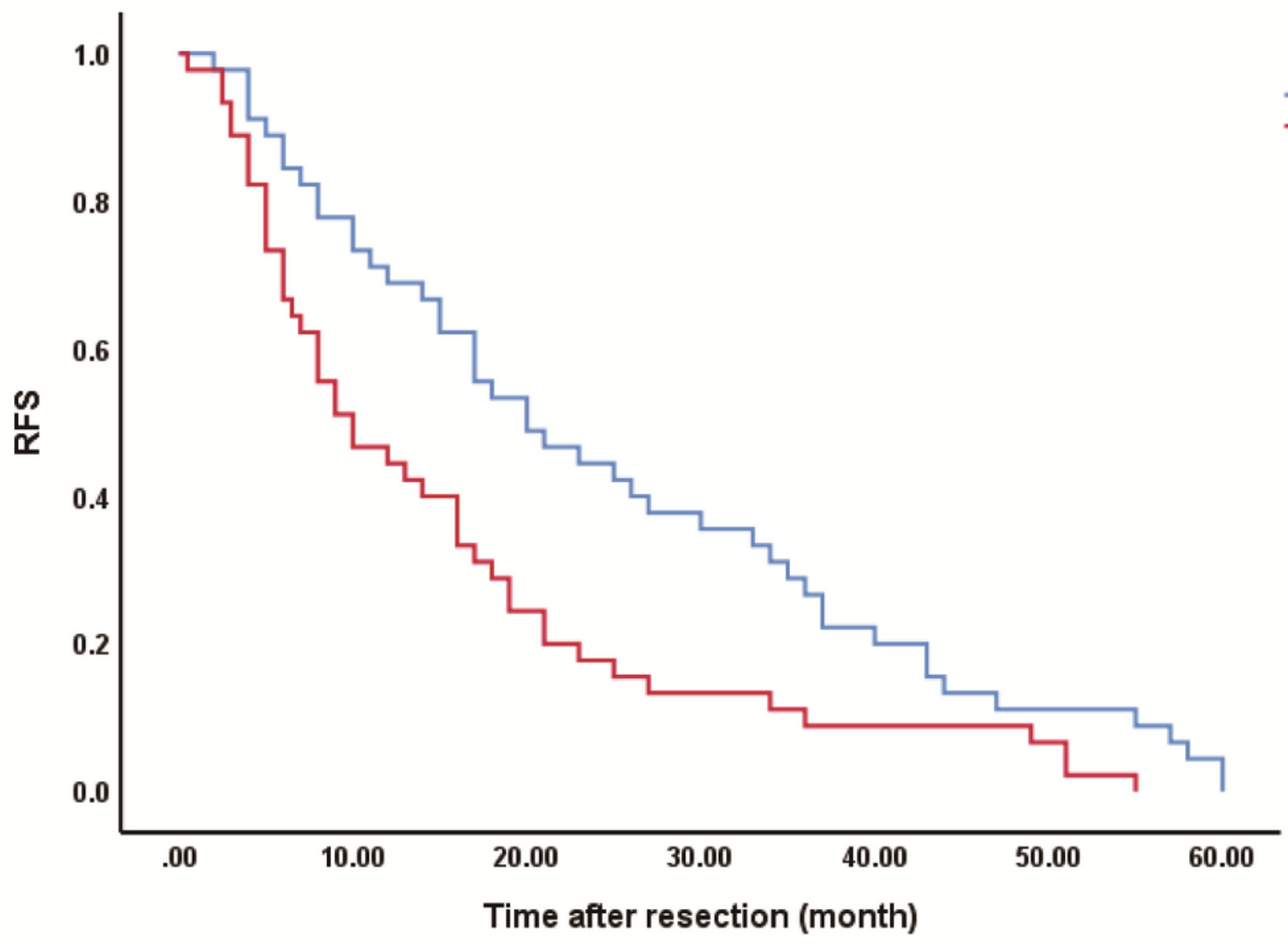

$\mathrm{P}=0.004$

\section{Figure 2}

Relapse-free survival (RFS) analysis in NP and TR.
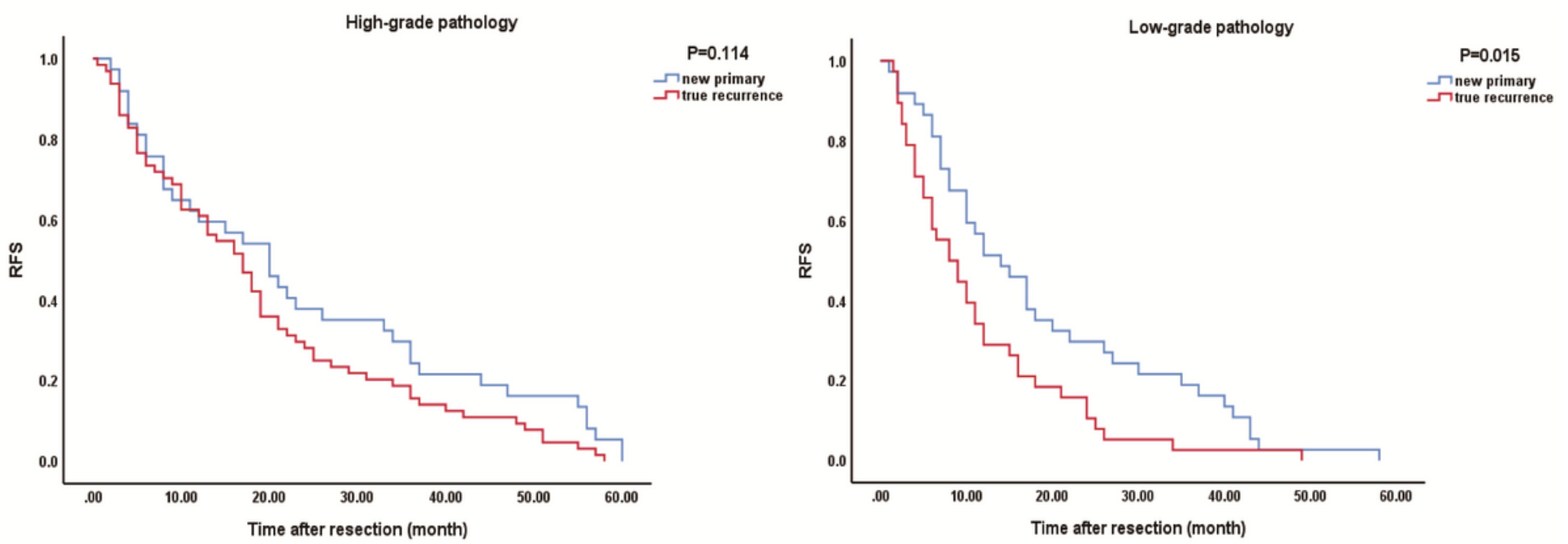

Figure 3

Relapse-free survival analysis (RFS) in low-grade and high-grade pathology. 

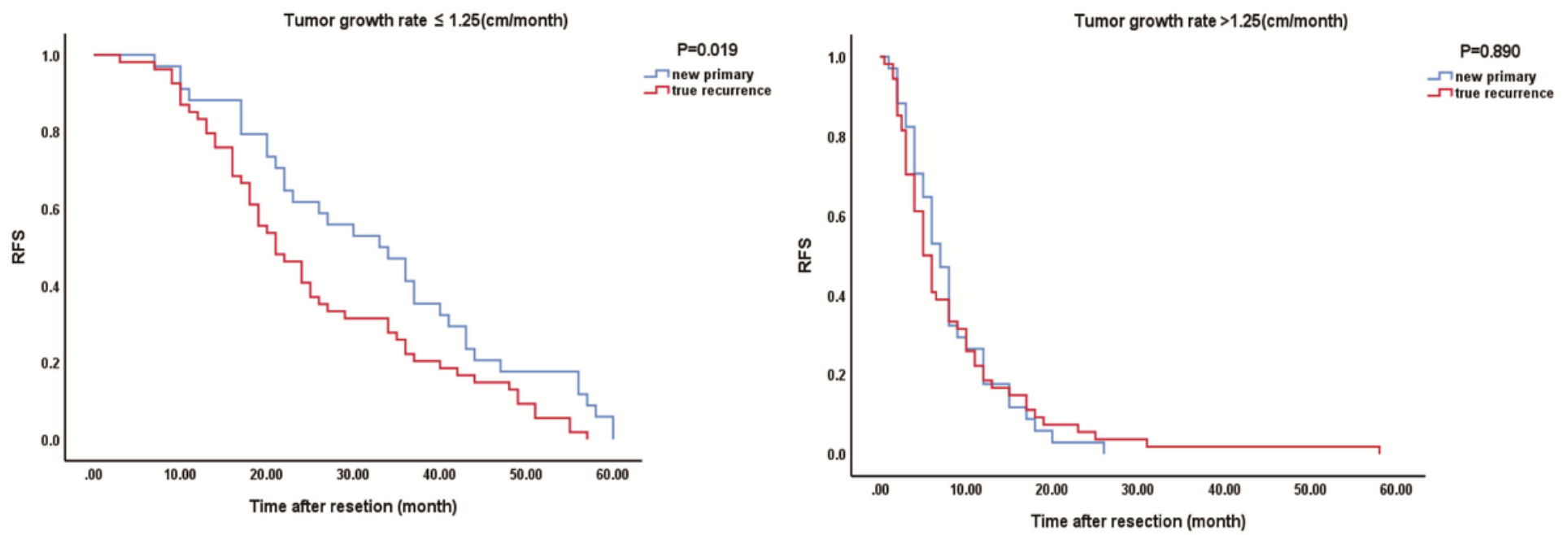

Figure 4

Relapse-free survival analysis (RFS) of the tumor growth rate $(1.25 \mathrm{~cm} /$ month is the median rate of 177 patients).
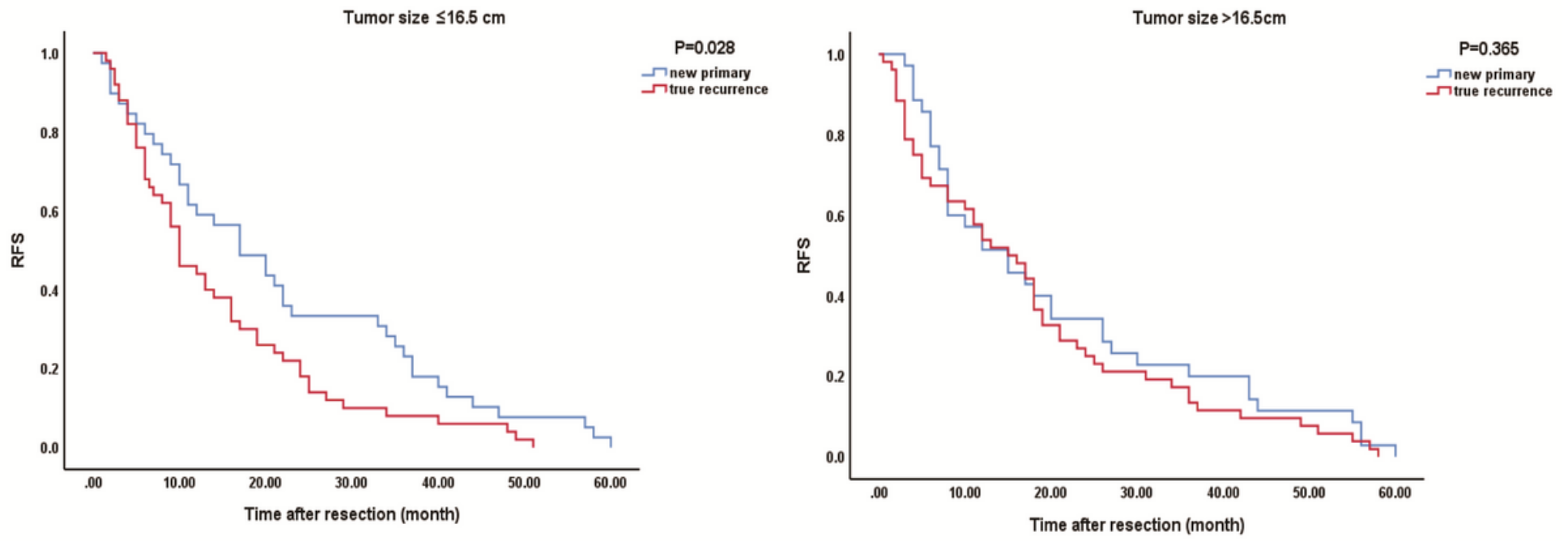

Figure 5

Relapse-free survival analysis (RFS) of tumor size $(16.5 \mathrm{~cm}$ is the median rate of 177 patients). 